\title{
STRATIFIED MAGNETICALLY DRIVEN ACCRETION-DISK WINDS AND THEIR RELATIONS TO JETS
}

\author{
Keigo Fukumura ${ }^{1,2}$, Francesco Tombesi ${ }^{2,3}$, Demosthenes Kazanas ${ }^{2}$, Chris Shrader $^{2,4}$, \\ EHUd BeHAR ${ }^{5}$, AND IOANNIS CONTOPOULOS ${ }^{6}$ \\ ${ }^{1}$ University of Maryland, Baltimore County (UMBC/CRESST), Baltimore, MD 21250, USA; fukumukx@jmu.edu \\ ${ }^{2}$ Astrophysics Science Division, NASA/Goddard Space Flight Center, Greenbelt, MD 20771, USA \\ ${ }^{3}$ Department of Astronomy, University of Maryland, College Park, MD 20742, USA \\ ${ }^{4}$ University Space Research Association, 10211 Wincopin Circle, Suite 620, Columbia, MD 21044, USA \\ ${ }^{5}$ Department of Physics, Technion, Haifa 32000, Israel \\ ${ }^{6}$ Research Center for Astronomy, Academy of Athens, Athens 11527, Greece \\ Received 2012 July 11; accepted 2013 October 31; published 2013 December 17
}

\begin{abstract}
We explore the poloidal structure of two-dimensional magnetohydrodynamic (MHD) winds in relation to their potential association with the X-ray warm absorbers (WAs) and the highly ionized ultra-fast outflows (UFOs) in active galactic nuclei (AGNs), in a single unifying approach. We present the density $n(r, \theta)$, ionization parameter $\xi(r, \theta)$, and velocity structure $v(r, \theta)$ of such ionized winds for typical values of their fluid-to-magnetic flux ratio, $F$, and specific angular momentum, $H$, for which wind solutions become super-Alfvénic. We explore the geometrical shape of winds for different values of these parameters and delineate the values that produce the widest and narrowest opening angles of these winds, quantities necessary in the determination of the statistics of AGN obscuration. We find that winds with smaller $H$ show a poloidal geometry of narrower opening angles with their Alfvén surface at lower inclination angles and therefore they produce the highest line of sight (LoS) velocities for observers at higher latitudes with the respect to the disk plane. We further note a physical and spatial correlation between the X-ray WAs and UFOs that form along the same LoS to the observer but at different radii, $r$, and distinct values of $n, \xi$, and $v$ consistent with the latest spectroscopic data of radio-quiet Seyfert galaxies. We also show that, at least in the case of $3 \mathrm{C} 111$, the winds' pressure is sufficient to contain the relativistic plasma responsible for its radio emission. Stratified MHD disk winds could therefore serve as a unique means to understand and unify the diverse AGN outflows.
\end{abstract}

Key words: accretion, accretion disks - black hole physics - galaxies: active - quasars: absorption lines - X-rays: galaxies

Online-only material: color figures

\section{INTRODUCTION}

Outflows are a common occurrence in accretion-powered objects. Their presence is affirmed by blueshifted absorption features in their optical, UV and X-ray spectra at frequencies of well defined transitions. Their velocities span a range of $10^{3}$, roughly between 100 and $100,000 \mathrm{~km} \mathrm{~s}^{-1}$, while the broad $\left(\sim 10,000 \mathrm{~km} \mathrm{~s}^{-1}\right)$ absorption troughs of UV transitions are the defining characteristic of the class of broad absorption line quasars (BAL QSOs). The launch of Hubble, ASCA, Chandra, $X M M-N e w t o n$, and Suzaku and their superior sensitivity, resolution, and energy bandwidth showed that $\simeq 50 \%$ of active galactic nuclei (AGNs) exhibit signatures of such outflows in their optical, UV and/or X-ray spectra. Of these, the high resolution long X-ray observations of Chandra and XMM-Newton are of particular interest because they discovered transitions that span a range of $\sim 10^{5}$ in ionization parameter, ${ }^{7} \xi$, (such as Fe I through Fe XXVI among others) and therefore sample a very broad range of conditions for the column density and velocity of plasma along the observers' line of sight (LoS). The fact that the plethora of these transitions spanning 5 decades in $\xi$ is squeezed within roughly 15 years of frequency underscores the utility of $\mathrm{X}$-ray spectroscopy.

\footnotetext{
$\xi \equiv L /\left(n r^{2}\right)$ where $L$ is an ionizing luminosity (usually defined between 1 and $1000 \mathrm{Ryd}), n$ is the plasma number density, and $r$ is distance from the ionizing source.
}

The first signature of X-ray absorbing plasma in AGN was that in the Einstein spectrum of the QSO MR 2251-178 (Halpern 1984), attributed to "warm" plasma of temperature $T \sim 10^{6} \mathrm{~K}$ (rather than "cool" $T \simeq 10^{4} \mathrm{~K}$ clouds), thus coining the term warm absorber (WA) for absorption features in the $\lesssim 1 \mathrm{keV}$ band. The ubiquity of WAs was eventually established by $A S C A$ which discovered absorption features of typical column density of $N_{\mathrm{H}} \sim 10^{20}-10^{22} \mathrm{~cm}^{-2}$ and $\xi \sim 10^{-1}-10^{4} \mathrm{erg} \mathrm{cm} \mathrm{s}^{-1}$ at moderate outflow velocities $\left(v \lesssim 3000 \mathrm{~km} \mathrm{~s}^{-1}\right)$ in the spectra of $\sim 50 \%$ of Seyfert 1 galaxies (Reynolds 1997; George et al. 1998). Following these discoveries, extensive spectroscopic observations with Chandra and XMM-Newton have been made to study in detail the physical conditions and spatial origin of WAs (e.g., Blustin et al. 2005; McKernan et al. 2007; Reeves et al. 2009a; Turner \& Miller 2009; Torresi et al. 2010, 2012). These are of interest because the presence of ionized absorbing gas of a very wide range of $\xi$ along the observers' LoS offers the opportunity to map the spatial distribution of this gas: one can easily see that the knowledge of $\xi$ (from the presence of certain ionic species) and the measurement of their hydrogen equivalent column $N_{\mathrm{H}}[\lesssim n(r) r]$ in the observed spectrum can provide a measure of the absorbing gas' density dependence on the distance from the continuum source along the observer's LoS. Such information is extremely valuable in assessing the global properties of the winds which presumably give rise to the observed absorbers.

A quantitative, more systematic formal way of implementing the above procedure is to construct the absorption measure 
distribution (AMD), namely the hydrogen-equivalent column of specific ions per decade of $\xi$; i.e., $\mathrm{AMD} \equiv d N_{\mathrm{H}} / d \log \xi$ (e.g., Holczer et al. 2007; Behar 2009; Detmers et al. 2011; Holczer \& Behar 2012, among others). These authors, instead of adding gas components at different values of $\xi$ and $N_{\mathrm{H}}$ until a satisfactory statistical significance $\chi^{2}$ is obtained, assumed a continuous dependence of binned $N_{\mathrm{H}}$ on $\xi$ (i.e., $d N_{\mathrm{H}} / d \log \xi \propto \xi^{s}$ ) and provided a global fit to the entire set of ionic transitions with $s$ as the sole parameter. The continuous dependence of $N_{\mathrm{H}}$ on $\xi$ also implies (assuming a smooth spatial gas distribution) a continuous dependence of the ionized medium density with $r$, its distance from the AGNs, of the form $n(r) \propto r^{-(2 s+1) /(s+1)}$. X-ray analysis of the spectra from a number of Seyfert galaxies showed that $N_{\mathrm{H}}$ has only a weak dependence on $\xi(s \simeq 0)$ (Behar 2009), implying a wind density profile of $n(r) \propto r^{-1}$ (the largest value of $s$ found was $s \simeq 0.3$ implying $\left.n(r) \propto r^{-1.25}\right)$. Furthermore, the presence of UV absorption features in the spectra of AGNs that exhibit X-ray WAs (e.g., Crenshaw et al. 2003) suggests a physical link between these components, which at present is not completely understood.

In parallel with the high-resolution observations discussed above, a number of lower resolution CCD X-ray observations of radio-quiet Seyferts and BAL QSOs have discovered absorption features identified as highly ionized iron (primarily $\mathrm{Fe}$ XXV/Fe XXVI at energies $\sim 7-8 \mathrm{keV}$ in the source frame) of column densities $\left(N_{\mathrm{H}} \sim 10^{23}-10^{24} \mathrm{~cm}^{-2}\right)$, generally higher than those of their moderately ionized ions at higher (blueshifted) velocities $(v / c \sim 0.1-0.7$ where $c$ is the speed of light), called ultra-fast outflows (UFOs; Tombesi et al. 2010a). These are seen across different AGN populations such as Seyferts, BAL QSOs and non-BAL QSOs (Chartas et al. 2003, 2007; Reeves et al. 2009b; Pounds \& Page 2006; Tombesi et al. 2010a, 2011a, 2012a). It is worth noting that similar features (UFOs) have also been reported in radio-loud Seyferts such as 3C 111 (e.g., Tombesi et al. 2010b, 2011b), indicating that these high outflow velocity transitions represent a generic feature of AGN rather than one associated with a specific AGN subclass. Furthermore, in the case of a BAL QSO APM 08279+5255, Chartas et al. (2009) noted a likely correlation between X-ray photon index $\Gamma$ and the measured outflow velocity of Fe Xxv, implying additional underlying physics pertaining to the velocities of these absorbers.

These observations of high ionization ( $\log \xi \gtrsim 4)$, high velocity $(v \gtrsim 0.1 c$ ) outflowing gas (UFOs) are of importance because they challenge the conventional radiative acceleration scenario of AGN outflows (e.g., Proga et al. 2000) which demands low ionization for this gas so that line radiation pressure driving is efficient; as such, they lend support to magnetic driving for these flows. The recent discovery of the UFOs in radio-loud Seyferts exhibiting jets (Tombesi et al. 2010b, 2011b) also seems to challenge a simplistic wind-jet dichotomy that associates "winds" with radio-quiet AGNs and "jets" with the radio-loud AGNs, perhaps implying a multicomponent gas in the same outflowing plasma across these broad AGN classes. While few simultaneous X-ray-radio studies having been conducted so far in radio-loud AGNs, Tombesi et al. (2012c, hereafter T12c) performed such a combined study of the $\mathrm{X}$-ray UFOs and the Very Long Baseline Array (VLBA) radio

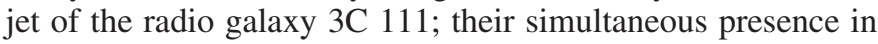
this object has indicated that these components can actually coexist in AGNs. Also, with respect to the coexistence of WAs and UFOs in radio-quiet Seyferts (e.g., Tombesi et al. 2010a, 2011a, 2012a, 2012b, hereafter T12b), have analyzed X-ray data from 35 radio-quiet Seyferts to obtain a number of correlations between $\xi, N_{\mathrm{H}}$, and $v$ of these components that argue in support of a single physical entity underlying the nature of WAs and UFOs.

Similar absorption features have been observed and studied intensively in binary systems including transient black hole (BH) candidates and neutron star low-mass X-ray binaries. These typically exhibit highly ionized plasma (e.g., $\mathrm{Si}, \mathrm{S}, \mathrm{Mg}$, Ar, and Fe) of $\log \xi \sim 3-4$ and $N_{\mathrm{H}} \sim 10^{21}-10^{22} \mathrm{~cm}^{-2}$ at typical outflow velocities ranging from $v \sim 300 \mathrm{~km} \mathrm{~s}^{-1}$ to $2000 \mathrm{~km} \mathrm{~s}^{-1}$, i.e., properties similar to those of AGNs but with lower velocities and more highly ionized species. Recently, however, King et al. (2012) found an extremely fast $(v / c \sim 0.03-0.05)$ ionized outflow based on the Fe XXV K-shell signature from Suzaku/XIS observations of a transient X-ray source, IGR J17091-3624. This is currently the fastest X-ray wind known in galactic binaries. This clearly illustrates a broad range of X-ray outflow kinematics possible around stellar-size compact objects. Some of the BH binaries, such as GRS 1915+105 (e.g., Neilsen \& Lee 2009; Neilsen et al. 2012) and H 1743-322 (e.g., Miller et al. 2006; Blum et al. 2010), exhibit relativistic radio jets akin to those of radio-loud Seyferts. In particular, these two components of outflows, X-ray absorbers and radio jets, appear to be anti-correlated: X-ray winds seem to be suppressed in the low/hard state of these objects which favor the presence of radio jets (e.g., Fender et al. 2004). Also, VLBA monitoring of jet emission combined with simultaneous $R X T E$ observations of low-frequency quasi-periodic oscillations seems to imply that the jet ejection is anti-correlated with the disk activity (see, e.g., Miller-Jones et al. 2012 for utilizing QPOs as a proxy of disk

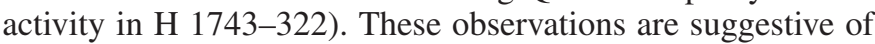
a mutual interplay between the disk (where X-ray outflows are also launched) and jet production.

In an effort to place the ensemble of these facts into the broader unifying context of accretion flows onto compact objects and their associated winds, Fukumura et al. (2010a, hereafter FKCB10a) have considered the photoionization of magnetohydrodynamic (MHD) accretion-disk winds. Motivated by the observed broad range of the WA and UFO ionization parameter and velocity range, they opted for self-similar models of MHD winds, as they naturally cover many decades in radius. Since their enunciation by Blandford \& Payne (1982, hereafter BP82), these winds have been the subject of many studies, both semi-analytical (BP82; Contopoulos \& Lovelace 1994, hereafter CL94; Königl \& Kartje 1994, hereafter KK94; Everett 2005; Ferreira 1997) and fully numerical (Proga 2003; Pudritz et al. 2006; Fendt 2006; Ohsuga et al. 2009; Porth \& Fendt 2010; Murphy et al. 2010). A crucial hint for the choice of a specific model has been the value of the parameter $s(\simeq 0)$ in the AMD relation (the dependence of $N_{\mathrm{H}}$ on $\xi$ ), since this determines the run of the wind density with distance. Clearly, observations favor density profiles close to $n(r) \propto 1 / r$, a value significantly different from that of the winds of BP82 (which imply $s \simeq 3 / 2$ ) and at significant odds with radiationdriven outlflows (which, for $\xi$ approaching its asymptotic value $\xi_{\infty}$, imply $s \gg 1$ and hence $\left.n(r) \propto r^{-2}\right)$. The important features of these density profiles are their almost constant (or slowly decreasing) column density per decade of radius and their linear decrease of $\xi$ with distance $r$, a fact that determines that high ionization ions have higher velocities than the lower ionization ones, in agreement with observations. The models of FKCB10a reproduce these features yielding velocities $v \sim 300 \mathrm{~km} \mathrm{~s}^{-1}$ for the moderately ionized species of Fe XVII 
and $v \sim 3000 \mathrm{~km} \mathrm{~s}^{-1}$ for the highly ionized ions of Fe XXVI. This work was extended by Fukumura et al. (2010b, hereafter FKCB10b), which demonstrated the model's broad applicability by successfully modeling the combined X-ray UFOs and UV broad absorption features of the BAL QSO APM 08279+5255 (Chartas et al. 2009); this was achieved by adjusting only the relative normalization of the $\mathrm{UV}$ and $\mathrm{X}$-ray fluxes to those appropriate for BAL QSOs.

To date, our publications on the subject (FKCB10a, FKCB10b), which presented the first attempt in this specific direction, have employed a single set of wind parameters implying an identical magnetic field geometry in both cases. The goal of the present work is to remedy this deficiency by an exploration of the wind geometry and ionization through a parameter search, keeping in mind the X-ray and radio AGN observations (T12b; T12c), with the underlying theoretical framework still based on the work of CL94. In Section 2 we summarize the basics of MHD accretion-disk wind models that have been previously applied for comparison with the observed X-ray WAs (FKCB10a) and UFOs (FKCB10b). In Section 3 we present our results and demonstrate a relevance of the outflows in a number of situations where those winds could provide a substantial impact on the surrounding environment, and we discuss the implications of our results in Section 4 with a comparison with BP82 winds in the Appendix.

\section{CHARACTERISTICS OF MAGNETIZED ACCRETION-DISK WINDS}

As is well known, the structure of axisymmetric MHD flows is determined by the magnetic flux function $\Psi(r, \theta)$ (see, e.g., CL94, Ferreira 1997; Vlahakis \& Königl 2003; Fendt 2006; Porth \& Fendt 2010). As discussed earlier, the broad range of the observed $\xi$ and $v$ of absorption features suggests a powerlaw form of all physical quantities with the radial coordinate $r$; as a result, we assume for $\Psi(r, \theta)$ the following separation of variables: $\Psi(r, \theta)$ in the power-law form of spherical radius $r$ as $\Psi(r, \theta) \equiv\left(r / r_{o}\right)^{q} \tilde{\Psi}(\theta) \Psi_{o}$, where $q$ is a self-similar index and $\Psi_{o}$ is the magnetic flux normalization at the innermost radius $r_{o}$ at which our scaling is assumed to be valid, and $\tilde{\Psi}(\theta)$, the angular dependence of this function which has to be determined numerically. We provisionally assume $r=r_{o} \equiv 3 R_{S}\left(R_{S}\right.$ is the Schwarzschild radius), the value of the innermost stable circular orbit of the Schwarzschild geometry. We make this choice based on the high velocities $(v \simeq 0.5 c$ ) observed in certain objects, with the understanding that those of our results pertaining to these innermost radii may not be accurate. However, we do not expect this assumption to affect the wind structure at the much larger scales covered by the observations.

With the above choice for $\Psi$ the magnetic field strength, velocity, number density, and total pressure take the following form

$$
\begin{gathered}
|\boldsymbol{B}(r, \theta)| \equiv B_{o}\left(\frac{r}{r_{o}}\right)^{q-2} \tilde{B}(\theta), \\
|\boldsymbol{v}(r, \theta)| \equiv v_{o}\left(\frac{r}{r_{o}}\right)^{-1 / 2} \tilde{v}(\theta), \\
n(r, \theta) \equiv \frac{1}{m_{p}}\left(\frac{B_{o}}{v_{o}}\right)^{2}\left(\frac{r}{r_{o}}\right)^{2 q-3} \tilde{n}(\theta), \\
p(r, \theta) \equiv K B_{o}^{2}\left(\frac{r}{r_{o}}\right)^{2 q-4} \tilde{n}(\theta)^{\Gamma},
\end{gathered}
$$

where

$$
\tilde{n}(\theta) \equiv \frac{F_{o}}{4 \pi} \frac{\tilde{B}_{p}(\theta)}{\tilde{v}_{p}(\theta)},
$$

$m_{p}$ is proton mass, and $K$ is the dimensionless polytropic constant related to plasma entropy. All the dimensionless angulardependent functions denoted by tildes must be numerically obtained from the conservation equations as the solution to the Grad-Shafranov equation with initial values on the disk at $(r, \theta)=\left(r_{o}, 90^{\circ}\right)$ to which all the quantities are normalized as denoted by the subscript "o." The magnetized wind is also characterized by its plasma $\beta$, defined as the ratio of thermal to magnetic field pressures

$$
\beta(r, \theta) \equiv \beta(\theta)=\frac{p(r, \theta) / K}{B(r, \theta)^{2}}=\frac{\tilde{n}(\theta)^{\Gamma}}{\tilde{B}(\theta)^{2}},
$$

where $\Gamma=5 / 3$ is the polytropic index of the wind plasma. We note that its LoS radial dependence drops out in our framework since $B(r) \propto r^{q-2}$ and $p(r) \propto r^{2 q-4}$.

Under steady-state axisymmetric conditions there are five conserved quantities along a streamline of given $\Psi(r, \theta)$; i.e., the particle flux to magnetic flux ratio $F$, the angular velocity of field lines $\Omega$, the total angular momentum of the plasma $H$, the total energy (Bernoulli function) $J$, and the entropy $S$ (see CL94 for details). $F$ and $H$ are defined as

$$
\begin{gathered}
F(\Psi) \equiv 4 \pi n m_{p} \frac{\left|\boldsymbol{v}_{p}\right|}{\left|\boldsymbol{B}_{p}\right|}, \\
H(\Psi) \equiv-F(\Psi) r v_{\phi}+r B_{\phi},
\end{gathered}
$$

where $\boldsymbol{v}_{p}$ and $\boldsymbol{B}_{p}$ are, respectively, the poloidal velocity and magnetic field while $v_{\phi}$ and $B_{\phi}$ are, respectively, the azimuthal velocity component and magnetic field strength.

We assume the disk threaded by the magnetic field to be infinitely thin, so we define boundary conditions at the point $\left(r=r_{o}, \theta=90^{\circ}\right)$ where $\boldsymbol{v} \equiv \boldsymbol{v}_{o} \sim \boldsymbol{v}_{\phi}$ with only a small component $v_{z, o}$ perpendicular to the disk surface $\left[v_{r}\left(90^{\circ}\right), v_{\theta}\left(90^{\circ}\right) \ll v_{\phi}\right]$. For a given set of $F_{o}$ and $H_{o}$, a transAlfvén wind solution can be obtained when the initial magnetic field line orientation $\theta_{o}$ is met with the regularity condition. ${ }^{8}$ With these constraints, $\Omega_{o}$ and $J_{o}$ are dependent quantities constrained by $F_{o}$ and $H_{o}$ (see CL94 and FKCB10a); i.e.,

$$
\begin{gathered}
\Omega_{o}=1-v_{\theta}\left(90^{\circ}\right)\left(F_{o}+H_{o}\right), \\
J_{o}=v_{\theta}^{2}\left(90^{\circ}\right)\left[1+\psi\left(90^{\circ}\right) 2\right] / 2-1 / 2-\Omega_{o},
\end{gathered}
$$

where $\psi\left(90^{\circ}\right)$ characterizes the angle the poloidal field/flow lines make with the disk at their footpoints (readers can easily verify that this angle is equal to $\tan ^{-1}\left\{q / \psi\left(90^{\circ}\right)\right\}$ with $v \phi\left(90^{\circ}\right)=1$ and $v_{\theta}\left(90^{\circ}\right)=0.01$ at the launching radius $r_{o}$ (see CL94 for details). We thus find that, for a given $q$, the wind structure is primarily governed by $F_{o}$ and $H_{o}$; the goal of the present study is to determine the structure and observable properties (i.e., column density, LoS velocity as a function of angle) of these winds and their relation

\footnotetext{
8 While physically valid winds must pass through a slow magnetosonic point we assume that the wind quickly becomes super-slow magnetosonic due to its efficient acceleration (see CL94 and Ferreira \& Pelletier 1995 in relation to the wind conditions).
} 
to observations. Specifically, the value of $F_{o}$ is generally responsible for determining the wind kinematics while $H_{o}$ plays an important role in shaping its poloidal structure. Note that one must adjust either $F_{o}$ or $H_{o}$ for a given initial poloidal angle of the magnetic field in order for winds to become super-Alfvénic (see the Appendix for a discussion on the viability for our choice of parameter sets). Although the other model parameters (see CL94 and FKCB10a) are also coupled to and affect the wind properties, we find them to be fundamentally less significant compared to $F_{o}$ and $H_{o}$.

The magnetic and velocity fields can be decomposed explicitly in poloidal and toroidal components as $\boldsymbol{B}(r, \theta) \equiv \boldsymbol{B}_{p}+\boldsymbol{B}_{\phi}$ and $\boldsymbol{v}(r, \theta) \equiv r \sin \theta \Omega \hat{\boldsymbol{\phi}}+m_{p} F /(4 \pi n) \boldsymbol{B}$, while the density at $\left(r_{o}, 90^{\circ}\right)$ can be explicitly expressed in terms of the dimensionless mass-accretion rate $\dot{m}$ (see FKCB10a) as $n_{o} \equiv n\left(r_{o}, 90^{\circ}\right) \equiv$ $\eta_{W} \dot{m} /\left(2 \sigma_{T} r_{s}\right)$, where $\eta_{W}$ is the ratio of the total mass flux rate launched in the wind to $\dot{m}$ (while the rest is accreted) and $\sigma_{T}$ is the Thomson cross section. Note also that in our discussion on density normalization that $\dot{m}$ always refers to the local mass flux at the innermost flow radius at $r \simeq r_{o}$ because the wind mass flux generally has a radial dependence. One can also calculate force elements in the wind (e.g., gas pressure force and magnetic forces) as $\nabla p_{\text {wind }} \equiv \nabla p_{\text {gas }}-(\boldsymbol{J} \times \boldsymbol{B}) / c$ (where $\boldsymbol{J}$ is the current density of the wind) which can be a measure of thermal equilibrium with ambient gas.

We now briefly discuss the issue of critical points in our winds. These are discussed in BP82, CL94, and also in Vlahakis et al. (2000) and Ferreira \& Casse (2004). A complete description would require that these solutions pass the fast and modified fast (i.e., the critical point in the direction perpendicular to that of the imposed symmetry) points as described in BP82 (and achieved in Vlahakis et al. 2000; Ferreira \& Casse 2004). However, these points lie at sufficiently high latitudes where the wind densities are very low and the gas ionization complete, so that they are effectively irrelevant to the absorption features observations. As a result, we limit our wind description to crossing the Alfvén and the slow-magnetosonic points. While crossing all critical points is an issue of principle with steady-state winds (e.g., BP82 and CL94 self-similar winds recollimate on the axis and even turn back), these points are in fact crossed without much trouble in nature; i.e., in time-dependent simulations (Pudritz et al. 2006; Fendt 2006; Porth \& Fendt 2010). Once the fluid has achieved escape velocity, it reaches infinity (albeit after a potential recollimation along the symmetry axis).

In the following calculations discussed in Section 3, therefore, we chose to stop the wind solutions past the Alfvén point arbitrarily to eliminate a physically less reliable portion of it. However, since our primary goal has only been to demonstrate the model's global viability with the observed X-ray absorbers and not to construct a comprehensive model of ultrarelativistic winds/jets, this approximation is justified up to near the Alfvén point. Most notably, we find that this manipulation makes little impact on our argument of WAs and UFOs in the context of MHD-driven outflows presented in this paper since most absorbers still lie within such an artificial cut-off.

\section{RESULTS}

Employing the framework discussed above, we have studied the structure and geometry of winds with two distinct density profiles, namely, winds with $n(r) \propto r^{-1}$ (i.e., $q \sim 1$; CL94, KK94), because they are favored by the X-ray absorber data, and winds with $n(r) \propto r^{-3 / 2}$ (i.e., $q \sim 3 / 4$; BP82), because
Table 1

Characteristics of Baseline MHD Wind Models

\begin{tabular}{lccccccccc}
\hline \hline Model & $n(r)$ & \multicolumn{1}{c}{$F_{o}$} & \multicolumn{1}{c}{$H_{o}$} & $\theta_{o}$ & $\Omega_{o}$ & $J_{o}$ & $\theta_{A}$ & $v_{p, 4} / v_{o}$ & $\theta_{\text {open }, 4}$ \\
\hline A & $r^{-1}$ & 0.065 & -1.7 & 1.276 & 1.016 & -1.51 & $38^{\circ}$ & 4 & $7^{\circ}$ \\
$\mathrm{B}$ & $r^{-1}$ & 0.03 & -2.455 & 1.872 & 1.024 & -1.52 & $53^{\circ}$ & 7 & $28^{\circ}$ \\
$\mathrm{C}$ & $r^{-1.5}$ & 0.05 & -3.152 & 5.7 & 1.032 & -1.53 & $76^{\circ}$ & 6 & $47^{\circ}$ \\
$\mathrm{D}$ & $r^{-1.5}$ & 0.1 & -1.68 & 2.105 & 1.015 & -1.515 & $59^{\circ}$ & 3 & $6^{\circ}$ \\
\hline
\end{tabular}

they are best known and most widely cited. Table 1 shows the functional form of $n(r)$, the values of $F_{o}, H_{o}, \Omega_{o}$, and $J_{o}$, the angle of the Alfvén surface $\theta_{\mathrm{A}}$, the wind asymptotic (i.e., at $z / r_{o}=10^{4}$ ) poloidal velocity normalized to $v_{o}, v_{\mathrm{p}, 4} / v_{o}$, and the wind opening angle $\theta_{\text {open, } 4}$ at $z / r_{o}=10^{4}$ for the wind element launched at the fiducial radius $r_{o}$. The corresponding poloidal wind structure of all four cases is shown in Figure 1 in linear scale in the computational domain $1-10^{4} r_{o}$. The figure shows the poloidal density distribution normalized to its maximum value $n_{o}=B_{o}^{2} /\left(m_{p} v_{o}^{2}\right)$ (in fact it shows the value of $\left.\log \left[m_{p} n /\left(B_{o} / v_{o}\right)^{2}\right]\right)$ along with its contours (solid thin lines), the wind streamlines $r(\theta)$ (solid thick lines) equally spaced by $\Delta r / r_{o}=10^{3}$, the poloidal velocity vectors $\boldsymbol{v}_{p} / v_{o}$ (gray arrows), and the velocity contours (dashed lines). A white solid line denotes the Alfvén surface where the plasma poloidal speed $v_{p}$ reaches the local Alfvén speed $v_{A} \equiv B_{p} / \sqrt{4 \pi m_{p} n}$. As mentioned earlier in Section 2, we decided to artificially truncate the super-Alfvén wind solution at a critical latitude $\theta_{c} \equiv \cot ^{-1}\left(100 \cot \theta_{A}\right)$ because of uncertainty in self-similarity as well as trans-fast nature of the solutions where $\theta_{A}$ denotes the latitude corresponding to the Alfvén point.

The values of variables in these figures in physical units can be obtained from Equations (1)-(6) with an appropriate normalization for the plasma density and velocity. While the velocity normalization can be obtained independently of the mass of the accreting object $M$, once a choice of the fiducial radius $r_{o}$ (close to the Schwarzschild radius $R_{S}$ ) is made (where $\left.v_{o} \simeq c\left(r_{o} / R_{S}\right)^{-1 / 2}\right)$, the normalization of the density (and the ensuing ones of the pressure and magnetic field) requires a value for the $\mathrm{BH}$ mass $M$ and the dimensionless accretion rate $\dot{m}$ (normalized to its Eddington value). The normalization of the density can be obtained by considering that for accretion or outflow at the local escape velocity and at the Eddington rate, the Thomson optical depth of the flow is equal to one or greater at $r_{o} \simeq R_{S}$; this implies that $n_{o} \simeq \dot{m} / \sigma_{T} R_{S}$ or $n_{o} \simeq 5 \times 10^{10}\left(\dot{m} / M_{8}\right) \mathrm{cm}^{-3}$. With these considerations and assuming magnetic field in equipartition with the kinetic energy density, i.e., $n m_{p} v_{o}^{2} / 2=B^{2} / 8 \pi$, in a flow with $\dot{m} \simeq 1$ and $M \simeq 10^{8} M_{\odot}$ we obtain the values

$$
\begin{aligned}
n(r, \theta) \simeq & 4.1 \cdot 10^{10}\left(\frac{B_{o}}{10^{4} \mathrm{G}}\right)^{2}\left(\frac{v_{o}}{0.4 c}\right)^{-2}\left(\frac{r}{r_{o}}\right)^{2 q-3} \\
& \times\left(\frac{\tilde{n}(\theta)}{\tilde{n}\left(90^{\circ}\right)}\right) \mathrm{cm}^{-3}, \\
\simeq & \times 10^{10}\left(\frac{\dot{m}}{M_{8}}\right)\left(\frac{r}{r_{o}}\right)^{2 q-3}\left(\frac{\tilde{n}(\theta)}{\tilde{n}\left(90^{\circ}\right)}\right) \mathrm{cm}^{-3} \\
p(r, \theta) \simeq & 2.1 \times 10^{4}\left(\frac{K}{0.01}\right)\left(\frac{B_{o}}{10^{4} \mathrm{G}}\right)^{2}\left(\frac{r}{r_{o}}\right)^{2 q-4} \\
& \times\left(\frac{\tilde{n}(\theta)}{\tilde{n}\left(90^{\circ}\right)}\right)^{\Gamma} \text { dyne } \mathrm{cm}^{-2},
\end{aligned}
$$



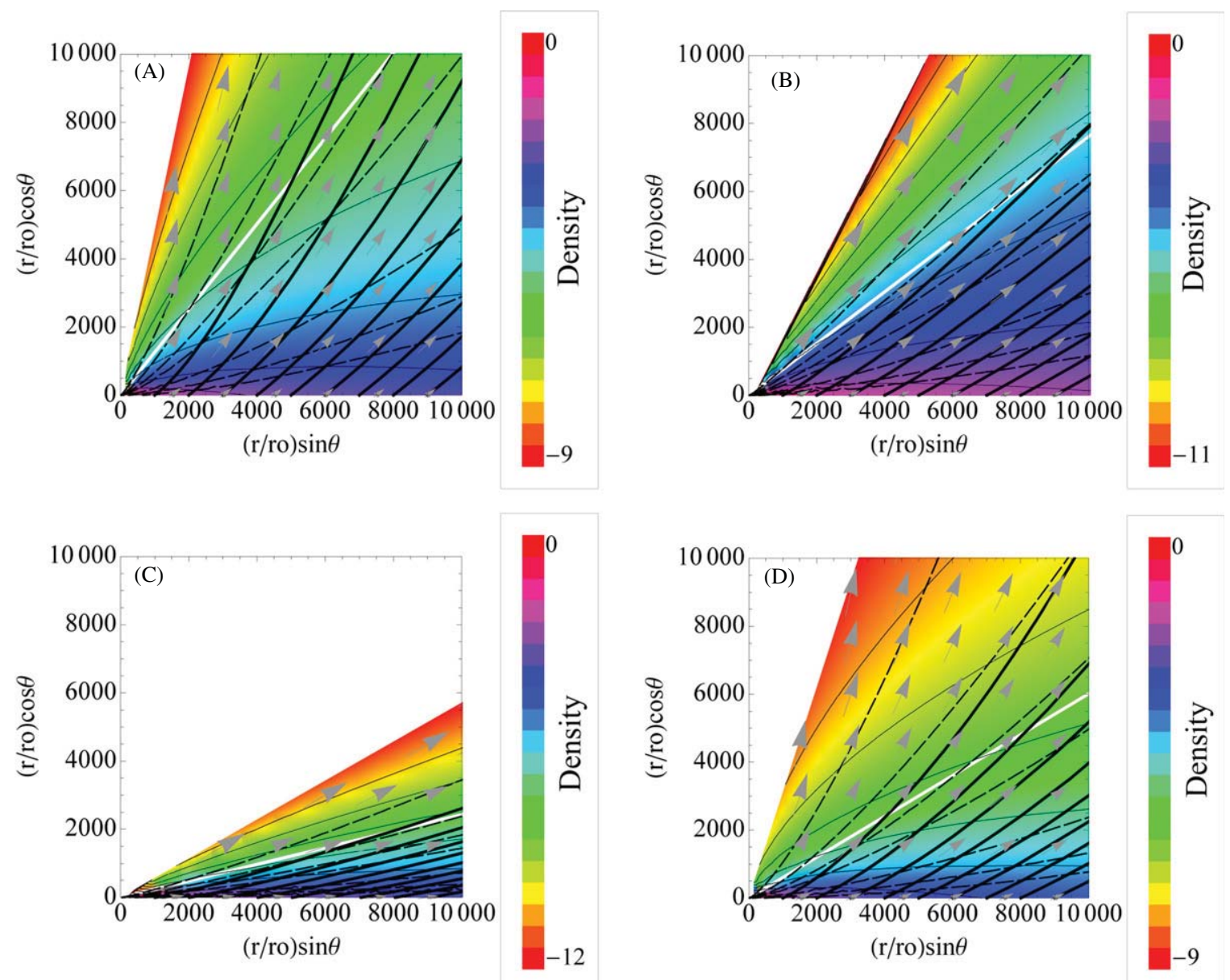

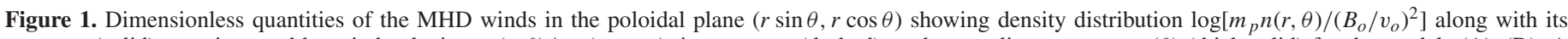

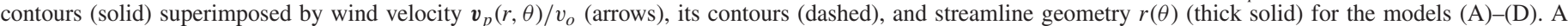

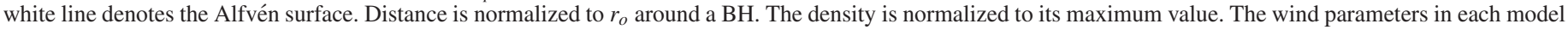
are listed in Table 1.

(A color version of this figure is available in the online journal.)

with the angular parts of $\tilde{n}, \tilde{p}$, and $\tilde{B}$ set equal to one on the disk surface $\left(\theta=90^{\circ}\right)$ and their $\theta$-dependence produced by the solution of the MHD equations. The normalization of the quantities given above, appropriate for $M=10^{8} M_{\odot}$, scales like $1 / M$, i.e., inversely with the $\mathrm{BH}$ mass, while the plasma $\beta$ is independent of it.

The properties of the models of Figure 1 are summarized in Table 1 (see the Appendix for a brief comparison with BP82 wind parameters). All these models have approximately the same values of $\Omega_{o}$ and $J_{o}$; models (A) and (B) have density profiles of $n(r) \propto r^{-1}$ (CL94; KK94), while models (C) and (D) have $n(r) \propto r^{-3 / 2}$ (BP82). The larger value of $H_{o}$ in model (B) results in less collimation, i.e., larger asymptotic opening angle $\left(\theta_{\text {open, } 4} \sim 28^{\circ}\right)$, compared with that of model (A) $\left(\theta_{\text {open, } 4} \sim 7^{\circ}\right)$ which has a smaller $H_{o}$ value. For the same reason, the Alfvén surface of (A) lies at smaller value of $\theta\left(\theta_{A} \sim 38^{\circ}\right)$ than of (B) $\left(\theta_{A} \sim 53^{\circ}\right)$. Finally, the combination of larger $H_{o}$ and smaller mass loading leads to larger asymptotic value in (B) $\left(v_{\mathrm{p}, 4} / v_{o} \sim 7\right)$ compared to that of $(\mathrm{A})\left(v_{\mathrm{p}, 4} / v_{o} \sim 4\right)$. The models with $n(r) \propto r^{-3 / 2}(\mathrm{C}, \mathrm{D})$ have relative dependencies similar to those of (A, B) and are summarized in Table 1. Finally, for models with similar values of $\Omega_{o}, J_{o}$, and $H_{o}$ but different density profiles, namely (A) and (D), while the asymptotic opening angles at $z=10^{4} r_{o}$ are similar, the Alfvén angle is smaller in (A) than (D) presumably as the result of the different poloidal current distribution of these two solutions via a coupling effect between $F_{o}$ and $H_{o}$.

These results are summarized in Figure 2(a) which shows the outflow opening angle $\theta_{\text {open }}$ along the innermost streamline, originating at $r=r_{o}$ (these curves correspond to the innermost streamlines in Figure 1) as a function of axial distance $z / r_{o}$ for models (A)-(D) with dots denoting the Alfvén points. The effects of the higher $H_{o}$ in models (B) and (C) are apparent there.

Our interest in the driving mechanism focuses primarily on the $J \times B$ force while not discussing the centrifugal force in detail. In the wind model considered here, toroidal wind velocity scales as $v_{\phi} \propto r^{-1 / 2}$ along a given LoS. Therefore, centrifugal force term can be written as $\sim \rho v_{\phi}^{2} / r \propto r^{-3}$ along the LoS and magnetic force term also can be written as $\nabla B^{2} \propto r^{-3}$ for $n \propto r^{-1}$. The relative strength of the centrifugal term can be dominant at smaller radii at least within the Alfvén surface. This behavior is indeed confirmed in Figure 2(b), which compares the force components tangent to the poloidal field line (i.e., accelerating components) following Lovelace \& Romanova (2013). The relative strength of each force component and its profile is consistent with those in other works (e.g., Figure 9 in Porth \& Fendt 2010) in that the magnetic part can be the dominant component even at smaller radii. Lii et al. (2012) also 

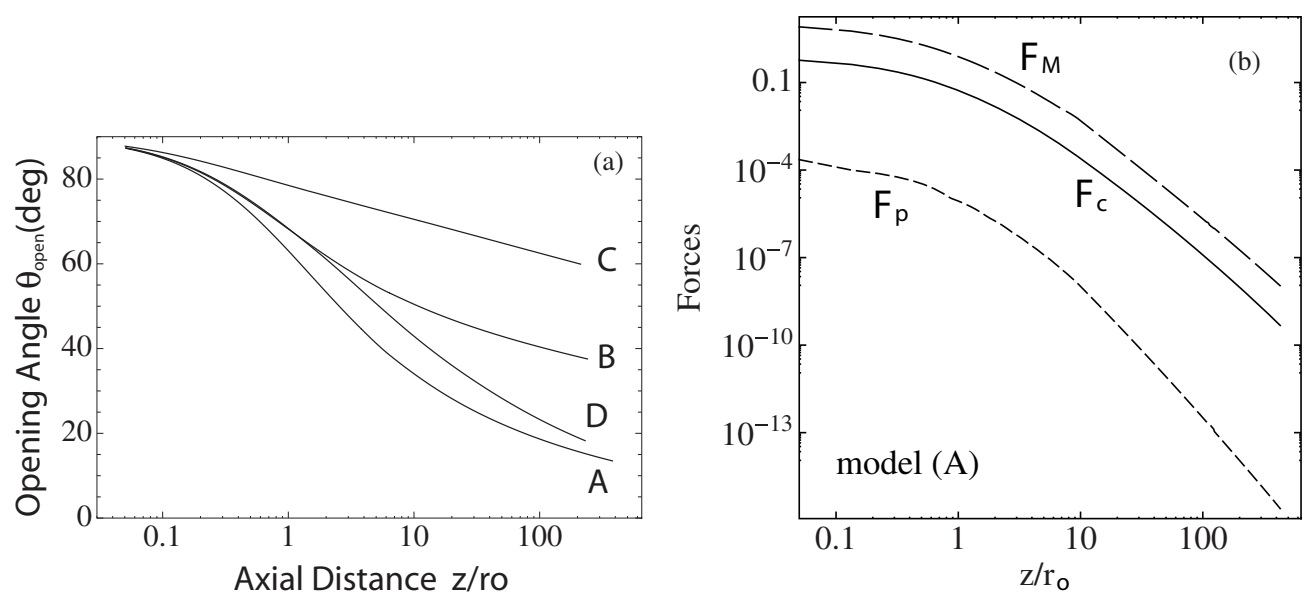

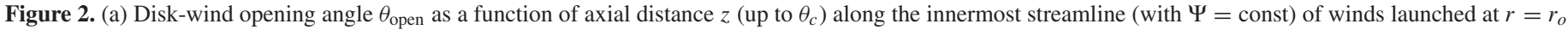

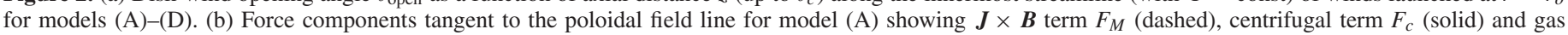
pressure term $F_{p}$ (dotted). The wind parameters are listed in Table 1.

argues in their 2.5D MHD jet simulations that the centrifugal force is cancelled by gravity and thus the jet is driven by a purely magnetic force. This mechanism is similar to the inner disk wind model discussed in Lovelace et al. (1991) and observed in simulations of conical winds in Romanova et al. (2009).

Focusing more on the global properties of magnetized wind, Figure 3 shows kinematic profiles $\boldsymbol{v}$ of the wind and the magnetic field $\boldsymbol{B}$ as well as the wind density profile $n$ as a function of axial distance $z / r_{o}$ for model (A) in the upper panels and (B) in the lower panels. Due to a geometrical difference between the two models, the radial wind velocity still exceeds the axial one in model (C). While velocity profiles $\boldsymbol{v}$ are independent of the models since we assume Keplerian boundary condition at the disk surface, the magnetic field profiles $\boldsymbol{B}$ and density $n$ are indeed dropping faster (i.e., $\propto z^{-3 / 2}$ ) in model (C) as expected. It is seen in both models that the wind undergoes a rapid acceleration phase from the base through the Alfvén point (i.e., the intersection between $v_{z}$ and $v_{A}$ ), after which the wind asymptotically approaches a coasting speed at $v_{\mathrm{p}, 4} / v_{o} \sim 4$ in model (A). The predominant initial orbital motion $\left(v_{\phi}\right)$ of the wind at the time of launch is found to be efficiently converted into axial motion $\left(v_{z}\right)$. These profiles follow $|\boldsymbol{v}| \propto z^{-1 / 2},|\boldsymbol{B}| \propto$ $z^{-1}$ and $n \propto z^{1 / 2}$ at large distances in consistence with the chosen $1 / r$ self-similarity in model (A).

As discussed in Section 1, the observations and physics of WAs mainly provide their ionization parameter $\xi$ and hydrogen equivalent column density $N_{\mathrm{H}}$, and, to the extent that can be measured, also the plasma velocity along the observer's LoS. Therefore, in order to relate the above models to observations, we plot in Figure 4 (in $\log -\log$ space) the structure of ionization parameter $\log \xi\left[\mathrm{erg} \mathrm{cm} \mathrm{s}^{-1}\right.$ ] for $\dot{m}=0.1$, along with the normalized density contours (dotted curves for $10^{-1}, 10^{-2}, 10^{-3}, 10^{-4}, 10^{-5}, 10^{-6}, 10^{-7}$ from bottom to top) and velocity contours (solid black curves for $v / c=0.5,0.3,0.1,0.05,0.03,0.01$ from innermost to outermost) of the same winds shown in Figure 1. Here, we only consider the geometrical dilution factor (see footnote 1), whereas in our photoionization calculations the opacity of the wind material was included (FKCB10a, FKCB10b). Because these quantities span a range of several decades, it is only reasonable to present the wind structure in logarithmic coordinates. Thus, color-coded $\log \xi$ is shown in the range $\sim 0$ and $\sim 10$. To make contact with observations, we also draw the observer LoS of different inclinations (diagonal gray lines corresponding to $\theta=10^{\circ}, 30^{\circ}, 45^{\circ}, 60^{\circ}, 80^{\circ}$ from top to bottom). One can now see that low inclination lines of sight intercept mainly high ionization and low column plasma (the iso-density curves are more closely spaced at the lower inclination directions). It is also apparent that the wind is highly ionized near its innermost edge where the velocities are also higher but the column intercepted is lower. It is not clear whether these sections of the wind can be detected in actual observations. With increasing inclination the LoS samples lower values of $\xi$ and velocity $v$ and higher values of the column $N_{\mathrm{H}}$. This model therefore naturally associates the observed WAs with objects of sufficiently high inclination angles.

The two bottom panels of Figure 4 correspond to winds with $n(r) \propto r^{-3 / 2}$ (models C, D). These panels look quite similar to those of models (A) and (B) above them. The obvious difference is the more closely spaced iso-density contours due to the steeper density profile; as a result, most of the column along a given LoS is concentrated near $\log (r)=0$. For the cases of both density profiles, we see that the higher angular momenta solutions leave a large fraction of the LoS not intercepting any plasma, at least within the computational domain. This fact would be of interest for the statistics of absorbers.

The column of these figures along a given LoS, one of the fundamental observables of the AGN absorption features, depends, as discussed above, on the value of (the normalized) mass flux and, as is apparent in Figure 4, on the winds' poloidal structure. Clearly models (B) and (C) require observer inclinations greater than $45^{\circ}$ to produce any significant absorption column. For model (A), a column of $N_{\mathrm{H}} \simeq 10^{23} \mathrm{~cm}^{-2}$ and velocity $\sim 10,000-15,000 \mathrm{~km} \mathrm{~s}^{-1}$, consistent with the observations of UFOs, assuming mass flux $\dot{m} \simeq 1$, demands an inclination angle $\theta \simeq 30^{\circ}-45^{\circ}$, based on the density function $n(\theta)$ of FKCB 10a, while the observed velocity implies $r / r_{o} \simeq(v / c)^{-2} \simeq 10^{3}$. In model (A) we see that in this region of parameter space $\log \xi \simeq 3-4$, consistent with the presence of an Fe XXV UFO. Clearly models (B) and (C) have very little column for inclinations $\theta \lesssim 30^{\circ}$. These figures also allow one to estimate the the velocity of a given ion (i.e., a given value of $\xi$ ) at a specific LoS and as such they are of value in setting a particular set of observations within the framework of these winds.

Besides the above connection between WAs and UFOs, we would further like to point out that the highly ionized 

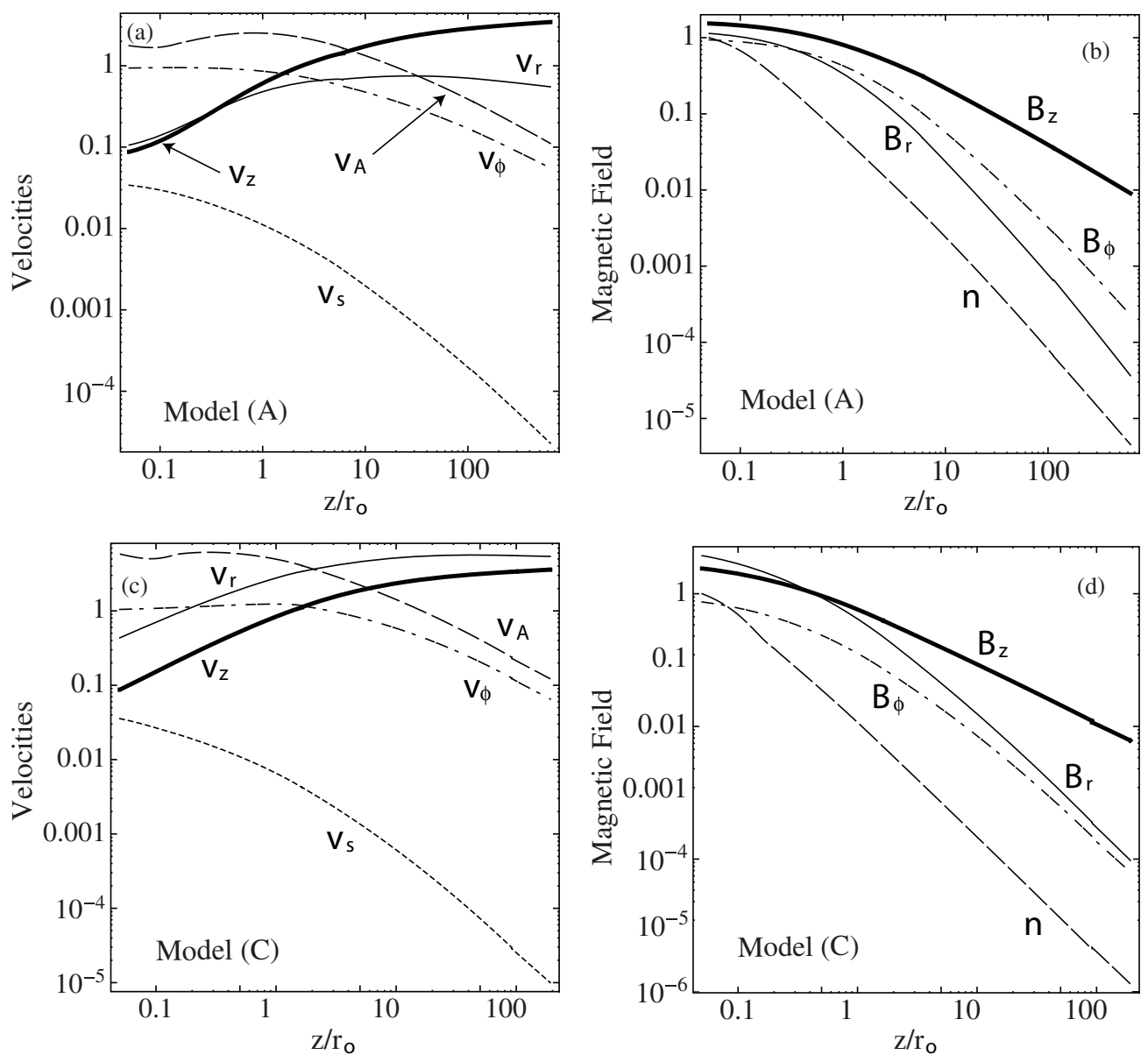

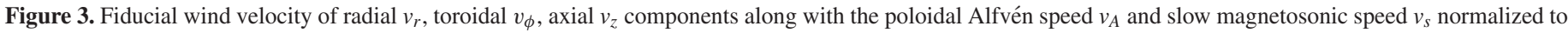

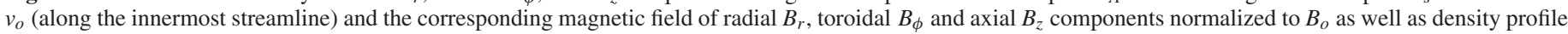

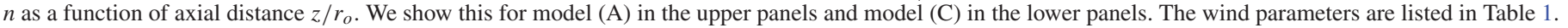

section of the wind near its axis provides thermodynamic characteristics consistent with radio jet properties. To this end, we show in Figure 5(a) (in linear space again), the innermost $\left(r \lesssim 800 r_{o}\right)$ structure of model (A): the contours of wind poloidal velocity $v_{p} / v_{o}$ are given by the solid curves (for $v_{p} / c=0.2,0.1,0.05,0.03,0.01$ from innermost to outermost) while those of the wind pressure by the dashed ones (for $p_{\mathrm{UFO}}=10^{-1}, 10^{-2}, 10^{-3}, 10^{-4}$ dyne $\mathrm{cm}^{-2}$ from bottom to top) and are superimposed on the color coded ionization structure $\log \xi(r, \theta)$, normalized to its maximum value. In this region, the pressure $p\left(200 r_{o}, 40^{\circ}\right) \sim 0.012$ dyne $\mathrm{cm}^{-2}$ and velocity $v_{p} / c \gtrsim 0.01$ are consistent with those obtained for the UFOs of the radio galaxy $3 \mathrm{C} 111 p \gtrsim 0.001-0.01$ dyne $\mathrm{cm}^{-2}$ (T12c), which were also shown to be compatible with the pressure of radio jets in the same region. Because of their tighter collimation, we would therefore associate models (A) and (D) with the structure of radio-loud Seyferts. Finally, a choice between these two models will rely on the dependence of the value of $N_{\mathrm{H}}$ on the ionization parameter $\xi$, assuming that the entire X-ray spectrum is produced by a point-like source, i.e., a source whose extent does not depend on the X-ray energy.

\section{SUMMARY AND DISCUSSION}

We have presented above the two-dimensional ionization structure of the MHD winds which our previous works we have associated with the WAs of AGNs. We have argued further that the same winds are also compatible with the more recently discovered UFOs and that in fact these features are different facets of the same underlying phenomenon, which apparently also occurs in radio galaxies, such as $3 \mathrm{C} 111$. We have also argued that the radio/X-ray observations of this later object imply pressure equilibrium between its radio jet emitting plasma and the plasma associated with its UFOs, suggesting that the former occupies, in pressure equilibrium, the near-axis region of the self-similar UFO-WA flows of our models.

The main results of the present work are encapsulated in Figures 1 and 4: they present the extreme cases of wind collimation geometry compatible with our assumptions for two different values of the wind radial density dependence. We found that while the density profile does affect the wind collimation, the latter is determined mainly by the value of their specific angular momentum $H_{o}$, with higher values producing significantly less collimation than lower ones. The issue of jet/ MHD wind collimation is of interest in studies of AGN statistics, considering that the outer regions of these winds could play the role of the AGN unification "torus" (Königl \& Kartje 1994). Thus, higher collimation implies a larger fraction of sources with X-ray absorption features. A more collimated flow due to less ambient matter entrainment, is more likely to produce a well defined, large-scale narrow jet and is therefore of higher velocity. Similarly, less collimated flows are likely to entrain more matter leading to lower velocity jets. The point of the present work is to note that, while it is difficult to image the regions 

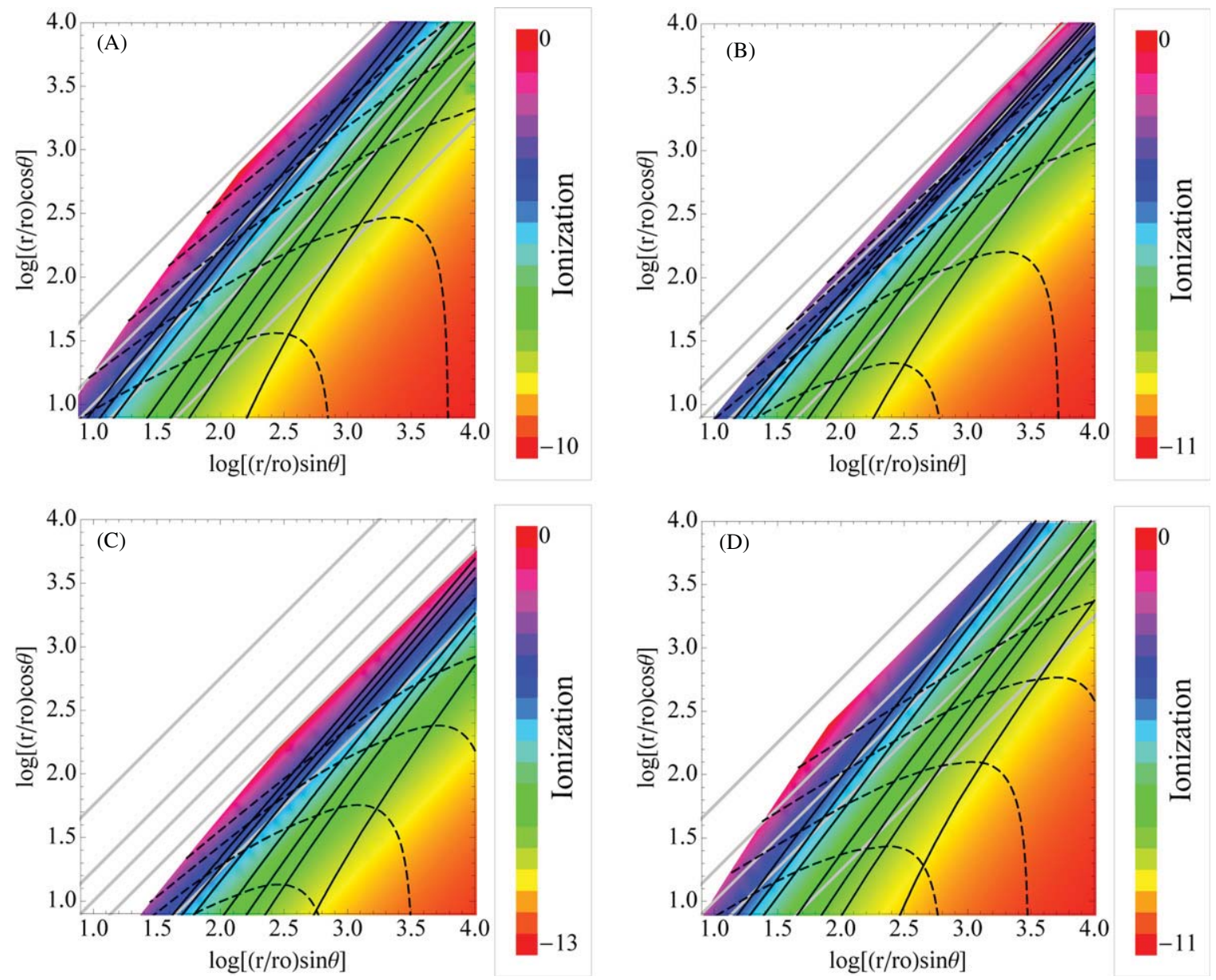

Figure 4. Contour plot (in log-log space) of models (A)-(D) corresponding to Figure 1 showing LoS poloidal velocity (solid) of $v / c=0.5,0.3,0.1,0.05,0.03,0.01$ (decreasing outward) and the density (dashed) $\log n=-1,-2,-3,-4,-5,-6,-7$ (decreasing upward) superimposed by color-coded ionization parameter $\log \xi$ which is normalized to their maximum value for $\dot{m}=0.1$. Five LoS angles are denoted by thick gray lines $\left(\theta=10^{\circ}, 30^{\circ}, 45^{\circ}, 60^{\circ}, 80^{\circ}\right.$ from top to bottom). The wind parameters are listed in Table 1.

(A color version of this figure is available in the online journal.)
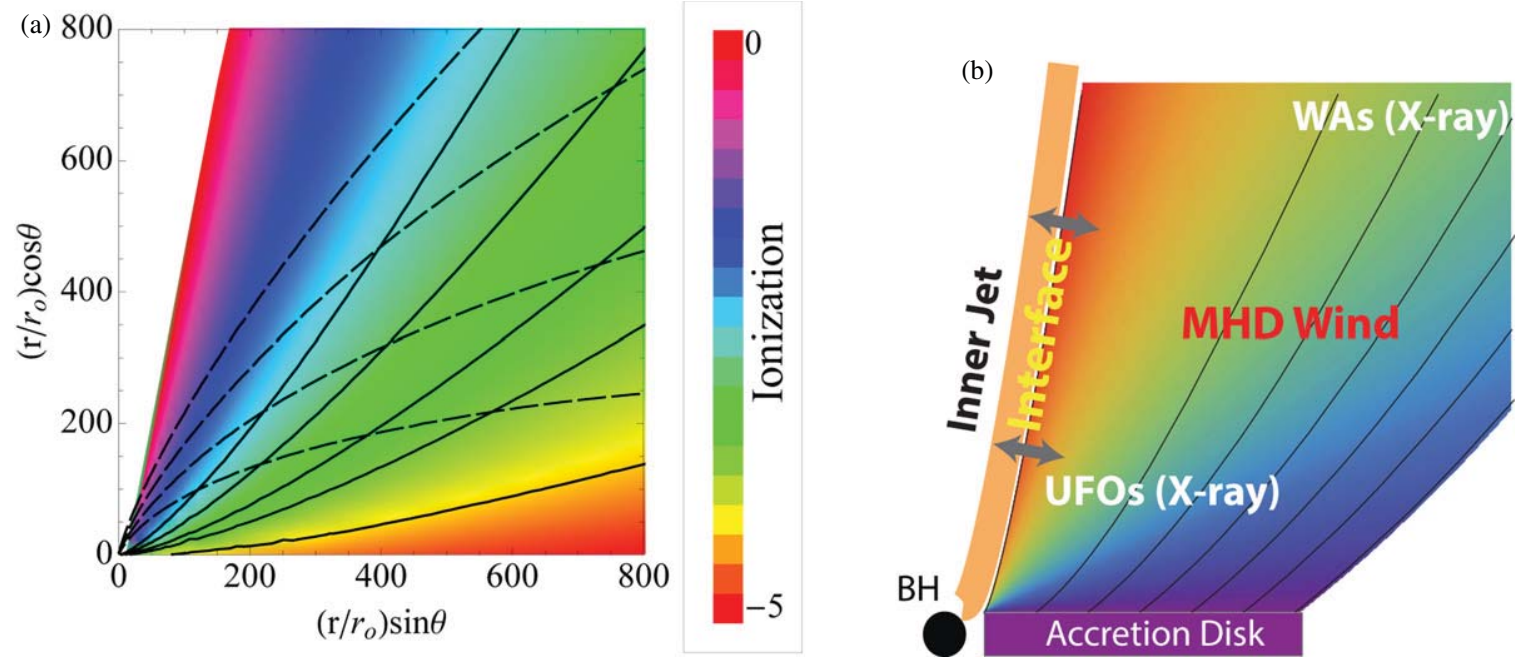

Figure 5. (a) Contour plot of model (A) showing LoS poloidal velocity (from top to bottom in solid) of $v / c=0.2,0.1,0.05,0.03,0.01$ and wind pressure (from top to bottom in dashed) of $p_{\mathrm{UFO}}=10^{-1}, 10^{-2}, 10^{-3}, 10^{-4}$ dyne $\mathrm{cm}^{-2}$ superimposed on color-coded ionization parameter log $\xi(r, \theta)$ that is normalized to its maximum value. (b) A schematic description of stratified MHD-driven wind structure manifesting WAs and UFOs (not to scale) with column distribution (similar to Figure 8 in $\mathrm{K} 12$ and Figure 5 in T12b). The arrows indicate a mutual interaction between inner relativistic jets and the MHD winds at their interface.

(A color version of this figure is available in the online journal.) 
where these flows originate and collimate, one can obtain their physical conditions (column, velocity) from spectroscopic Xray observations and also its collimation properties from the statistics of AGNs with a given amount of obscuration (Malizia et al. 2009; Terashima 2010) and their relation to the direction of a larger scale radio jet. A resolution of these issues, as well as those of systematics such as the obscuration dependence on luminosity (Tueller et al. 2008; Bourlon et al. 2011), require further observations and modeling (Proga 2003; Everett 2005; Kazanas et al. 2012). However, we believe that large-scale MHD winds will form the basis for an account of such global properties. Figure 5(b) illustrates a proposed manifestation of apparently distinct absorbers in the MHD wind discussed in this study. While it is beyond the scope of this work, we also note in a global viewpoint that the radial extent of magnetized winds is coupled to the disk magnetization (Murphy et al. 2010).

In addition, there is an issue of the radial density dependence of these winds. As we discussed in the Introduction, the main motivating factor of our previous work, have been the observations of Behar (2009) that indicate $N_{\mathrm{H}} \propto \xi^{s}$ where $s \simeq 0$ and imply a LoS density profile $n(r) \propto r^{-(2 s+1) /(s+1)}, 0<s<1$ with most values closer to $s \simeq 0$ (the parameter $s$ is related to that $q$ of Equation (1) by $2 q=(s+2) /(s+1))$. As we noted, this scaling suggests correlations between the column $N_{\mathrm{H}}$, the velocity $v$ and the ionization parameter $\xi$ of specific transitions at a given object (for instance $N_{\mathrm{H}} \propto v^{2 s /(s+1)}, v \propto$ $\left.\xi^{(s+1) / 2}\right)$. More recently, observations, mainly of UFOs but also certain WA transitions, in an ensemble of (rather than a single) AGNs (Tombesi et al. 2010b, 2012a, T12c), have shown on the aggregate a preference for the value $s \simeq 1$, implying a LoS density profile closer to $n(r) \propto r^{-3 / 2}$. At this point it would be of interest to note that models that include a combined accretion-outflow approach to the problem (Ferreira 1997; Ferreira \& Casse 2004) are more restricted in their choice of parameters than those presented above and do provide additional restrictions to the values of $s$. So, "cold" flows (Ferreira 1997) are restricted to density profiles very close to $n(r) \propto r^{-3 / 2}$, while models with "entropy injection" near the Alfvén surface (Ferreira \& Casse 2004) can yield density profiles close to $n(r) \propto r^{-1}$. At this point we will reiterate that $\mathrm{X}$-ray spectroscopy observations could provide the distinction between these extreme cases thus establishing a probe of the accretion-outflow physics of these systems.

Another issue with the solutions we present is their reliance on self-similarity. The utility of this technique is not only mathematical, i.e., in allowing one to obtain solutions of the MHD equations; it is of importance because it provides solutions that span a multiple decades in radius. Solutions of this type are demanded by the data, i.e., the large range in ionization parameter spanned by the observed X-ray transitions, as discussed in Section 1. Whether the simple functional form of the model parameters given above extends over the entire domain of validity of these solutions is an issue that should be answered observationally. Deviations from a single power law would indicate the presence of additional physics that will have to be included in such calculations.

By their nature, self-similar solutions cannot cover the region near the flow symmetry axis and they must also terminate at some distance from the origin. As we have noted above, the near-axis high latitude region is much less dense and much more highly ionized than the lower latitude ones, so from the interpreting observed point of view of absorption features which is the main thrust of the present paper, it is not of significance. It is certainly of significance mathematically and for impacting the physics of jets, as the magnetic fields of this region are likely to thread the $\mathrm{BH}$ event horizon. Pressure balance requires that the pressure there match that at larger radii where the self-similar solutions are valid. This is possibly the pressure of relativistic particles that gives rise to the radio jets observed. Such models that combine a self-similar disk-wind solution with an MHD flow of limited lateral extent (i.e., one in which the magnetic field threads a star or a $\mathrm{BH}$ ), have recently appeared in the literature (Matsakos et al. 2008, 2009; Porth \& Fendt 2010). Again, it is very conceivable that the winds may consist of two components; an axial collimated jet of low matter density probably originating from the $\mathrm{BH}$ itself surrounded by an extended disk wind (e.g., Ferreira et al. 2010) also similar to our view depicted in Figure 5(b). It is of interest to note that the last of the above references finds flows with opening angles between $4^{\circ}$ and $6^{\circ}$, which is not very different of those of models (A) and (D).

At large radii, these solutions cannot be trusted beyond an equatorial distance larger than that of the underlying disk. This distance is not known. However, it cannot be larger than the $\mathrm{BH}$ influence radius, $R_{B} \sim M_{\mathrm{BH}} / \sigma^{2}$, i.e., the distance at which the disk Keplerian velocity matches the velocity dispersion $\sigma=\left\langle v^{2}\right\rangle^{1 / 2}$ of the AGN surrounding spheroid. However, given the relation between $M_{\mathrm{BH}}$ and $\sigma$, namely $\left(M_{\mathrm{BH}} / 10^{8} M_{\odot}\right) \sim$ $\left(\sigma / 200 \mathrm{~km} \mathrm{~s}^{-1}\right)^{4}$ (Ferrarese \& Merritt 2000), this distance is of the order of $R_{B} \sim 10^{19}\left(M_{\mathrm{BH}} / 10^{8} M_{\odot}\right)^{-1 / 2} \sim 10^{19} M_{8}^{-1 / 2} \mathrm{~cm}$. It is of interest to note that absorption feature observations, in conjunction with our models, could provide an independent estimate of this distance and therefore an independent measure of the $M-\sigma$ relation.

Finally, one should bear in mind that density profiles $n(r) \propto$ $r^{-\alpha}$ produce winds whose mass flux increases with distance for $\alpha \leqslant 3 / 2$. Applying the profile with $\alpha=1$ and $\dot{m}=0.1$ used in our models to an accreting BH of $M=10^{8} M_{\odot}$, we obtain a total mass flux of

$$
\begin{aligned}
\dot{M}_{\text {out }}^{\text {(global) }} \equiv & m_{p} \int_{z=z_{c}} d^{2} x n(r, \theta) v_{z} \sim \frac{4 \sqrt{2} G^{2}}{c^{5}} F B_{o}^{2} M^{2} r_{\text {out }}^{1 / 2} \\
\sim & 6.5 M_{\odot}\left(\frac{F}{0.1}\right)\left(\frac{B_{o}}{10^{4} \mathrm{G}}\right)^{2}\left(\frac{M}{10^{8} M_{\odot}}\right)^{2} \\
& \times\left(\frac{r_{\text {out }}}{10^{6} r_{o}}\right)^{1 / 2} \text { year }^{-1},
\end{aligned}
$$

a value much higher than that needed to power its bolometric luminosity of $\sim 10^{45} \mathrm{erg} / \mathrm{s}$. The corresponding (integrated) kinetic power ${ }^{9}$ is given by $\dot{E}_{\text {out }}^{\text {(local) }} \equiv \dot{M}^{\text {(local) }} v_{\text {out }}^{2} \propto r^{-1 / 2}$. Assuming $r_{\text {in }} / r_{o} \sim 100$, this yields $\dot{E}_{\text {out }}^{\text {(global) }} \sim 10^{44} \mathrm{erg} \mathrm{s}^{-1}$ that is indeed consistent with the estimated UFO power in $3 \mathrm{C} 111$ (Tombesi et al. 2010b, 2012a, T12c) providing a large impact on AGN feedback process at large scales. On the other hand, the cumulative power of UV/soft X-ray WAs may be able to reach this level as well (e.g., Crenshaw \& Kraemer 2012). It is interesting to note that outflow rates, estimated by $\mathrm{X}$-ray spectroscopy in BH binaries (Neilsen \& Lee 2009; Neilsen et al. 2012), were found to be much larger than the accretion rates needed to power their X-ray luminosity.

On a closing note, although in this work we primarily focused on physical conditions and a global geometrical structure of

\footnotetext{
9 Also see K12 for the discussion of radial dependence of the wind quantities.
} 
MHD winds, we are currently simulating the corresponding spectral signatures of the absorption lines within this model (K. Fukumura et al. 2013, in preparation). To constrain individual wind parameters (such as $F_{o}, H_{o}$, and their dependency on $\dot{m}$ and $\theta$ ), however, one may require numerical calculations of $\mathrm{X}$ ray absorption feature with sets of MHD parameters and perform a $\chi^{2}$-test in comparison with data; i.e., observationally constrain the wind parameters that would otherwise be inaccessible. While it is still challenging to constrain all the wind variables, one can still hope to restrict the range of wind parameters allowed by spectrum analysis. The capabilities of the upcoming mission Astro- $H$ should be instrumental in performing these insightful observations.

K.F. thanks an anonymous referee for improving the manuscript and M. Nakamura for his inspiring comments on the draft manuscript. E.B. is supported by grants from the Israel Science Foundation and from Israel's Ministry of Science and Technology.

\section{APPENDIX}

\section{A BRIEF COMPARISON OF OUR WIND PARAMETERS WITH BP82 WINDS}

We have computed and examined four magnetized wind solutions to steady-state, axisymmetric ideal MHD equations under Newtonian gravity (as in CL94, FKCB10a, FKCB10b, and K12) in the context of its potential role of outflow physics. Generic properties of MHD outflows considered in this study include mass loading and angular momentum as well as the global density profile. We suggest that smaller angular momentum tends to help collimate the outflow poloidal structure while a lower plasma-to-magnetic flux ratio generally leads to a more efficient wind acceleration by the action of a large-scale magnetic fields (at least predominantly relative to other mechanisms such as radiation pressure).

Here, we would like to show a viability of the sets of our wind parameters by recalling some of the fundamental definitions of the conserve quantities. As discussed in CL94, one can express $F_{o}$ in terms of some "known" quantities from Equation (7) combined with

$$
F(\Psi)=r_{\Psi}^{q-3 / 2} F_{o} \frac{B_{o}}{v_{o}},
$$

to obtain

$$
\begin{aligned}
F_{o} & =4 \pi \rho \frac{v_{p}}{B_{p}} \frac{v_{o}}{B_{o}} \\
& \sim 10^{11}\left(0.01 \times(0.7 c)^{2}\right) /\left(10^{4}\right)^{2} \sim \mathcal{O}\left(10^{-2}\right) .
\end{aligned}
$$

assuming a typical set of values (for AGNs) of $n_{o} \sim 10^{10} \mathrm{~cm}^{-3}$ (Crenshaw et al. 2003 and references therein), $B_{p} \sim B_{o} \sim$ $10^{4} \mathrm{G}$, and $v_{p} \sim 0.01 v_{o}$ at the innermost launching radius. This value is in consistence with our chosen values. For the rest of the parameters, we (this paper and CL94) are also convinced of consistency with our parameter sets in comparison with BP82 winds as also discussed in CL94; i.e., one can make a one-toone mapping between BP82 wind parameters and ours by noting that

$$
\lambda \leftrightarrow-H_{o} / F_{o},
$$

where BP82's characteristic value of $\lambda=30$ leads to $-H_{o} \sim$ $\mathcal{O}(1)$. From our boundary condition at the disk surface expressed in Equation (9) (see also CL94) one finds

$$
\Omega_{o}=1-\left(F_{o}+H_{o}\right) V_{z, 0} \sim \mathcal{O}(1)
$$

where we have used $F_{o}$ and $H_{o}$ above together with $V_{z, 0} \sim 0.01$. Therefore, we are confident in our parameter choice.

\section{REFERENCES}

Behar, E. 2009, ApJ, 703, 1346

Blandford, R. D., \& Payne, D. G. 1982, MNRAS, 199, 883 (BP82)

Blum, J. L., Miller, J. M., Cackett, E., et al. 2010, ApJ, 713, 1244

Blustin, A. J., Page, M. J., Fuerst, S. V., Branduardi-Raymont, G., \& Ashton, C. E. 2005, A\&A, 431, 111

Bourlon, D., Ajello, M., Greiner, J., et al. 2011, ApJ, 728, 58

Chartas, G., Brandt, W. N., \& Gallagher, S. C. 2003, ApJ, 595, 85

Chartas, G., Eracleous, M., Dai, X., Agol, E., \& Gallagher, S. C. 2007, ApJ, 661,678

Chartas, G., Saez, C., Brandt, W. N., Giustini, M., \& Garmire, G. P. 2009, ApJ, 706, 644 (C09)

Contopoulos, J., \& Lovelace, R. V. E. 1994, ApJ, 429, 139 (CL94)

Crenshaw, D. M., \& Kraemer, S. B. 2012, ApJ, 753, 75

Crenshaw, D. M., Kraemer, S. B., \& George, I. M. 2003, ARA\&A, 41, 117

Detmers, R. G., Kaastra, J. S., Steenbrugge, K. C., et al. 2011, A\&A, 534, 38

Everett, J. E. 2005, ApJ, 631, 689

Fender, R. P., Belloni, T. M., \& Gallo, E. 2004, MNRAS, 355, 1105

Fendt, C. 2006, ApJ, 651, 272

Ferrarese, L., \& Merritt, D. 2000, ApJL, 539, L9

Ferreira, J. 1997, A\&A, 319, 340

Ferreira, J., \& Casse, F. 2004, ApJL, 601, L139

Ferreira, J., \& Pelletier, G. 1995, A\&A, 295, 807

Ferreira, J., Petrucci, P. O., Murphy, G., Zanni, C., \& Henri, G. 2010, in ASP Conf. Ser. 427, Accretion and Ejection in AGN: A Global View, ed. L. Maraschi, G. Ghisellini, R. D. Ceca, \& F. Tavecchi (San Francisco, CA: ASP), 49

Fukumura, K., Kazanas, D., Contopoulos, I., \& Behar, E. 2010a, ApJ, 715, 636 (FKCB10a)

Fukumura, K., Kazanas, D., Contopoulos, I., \& Behar, E. 2010b, ApJL, 723, L228 (FKCB10b)

George, I. M., Turner, T. J., Netzer, H., et al. 1998, ApJS, 114, 73

Halpern, J. P. 1984, ApJ, 287, 90

Holczer, T., \& Behar, E. 2012, ApJ, 747, 71

Holczer, T., Behar, E., \& Kaspi, S. 2007, ApJ, 663, 799

Kazanas, D., Fukumura, K., Behar, E., Contopoulos, I., \& Shrader, C. R. 2012, AstRv, 7, 92 (K12)

Königl, A., \& Kartje, J. F. 1994, ApJ, 434, 446 (KK94)

King, A. L., Miller, J. M., Raymond, J., et al. 2012, ApJL, 746, L20

Lii, P., Romanova, M., \& Lovelace, R. 2012, MNRAS, 420, 2020

Lovelace, R. V. E., Berk, H. L., \& Contopoulos, J. 1991, ApJ, 379, 696

Lovelace, R. V. E., Romanova, M. M., \& Lii, P. 2013, arXiv:1306.1160

Malizia, A., Stephen, J. B., Bassani, L., et al. 2009, MNRAS, 399, 944

Matsakos, T., Massaglia, S., Trussoni, E., et al. 2009, A\&A, 502, 217

Matsakos, T., Tsinganos, K., Vlahakis, N., et al. 2008, A\&A, 477, 521

McKernan, B., Yaqoob, T., \& Reynolds, C. S. 2007, MNRAS, 379, 1359

Miller, J. M., Sivakoff, G. R., Altamirano, D., et al. 2006, ApJ, 646, 394

Miller-Jones, J. C. A., Raymond, J., Homan, J., et al. 2012, MNRAS, 421, 468

Murphy, G. C., Ferreira, J., \& Zanni, C. 2010, A\&A, 512, 82

Nielsen, J., \& Lee, J. C. 2009, Natur, 458, 481

Neilsen, J., Petschek, A. J., \& Lee, J. C. 2012, MNRAS, 421, 502

Ohsuga, K., Mineshige, S., Mori, M., \& Kato, Y. 2009, PASJ, 61, L7

Porth, O., \& Fendt, C. 2010, ApJ, 709, 1100

Pounds, K. A., \& Page, K. L. 2006, MNRAS, 372, 1275

Proga, D. 2003, ApJ, 585, 406

Proga, D., Stone, J. M., \& Kallman, T. R. 2000, ApJ, 543, 686

Pudritz, R. E., Rogers, C. S., \& Ouyed, R. 2006, MNRAS, 365, 1131

Reeves, J. N., O'Brien, P. T., Braito, V., et al. 2009b, ApJ, 701, 493

Reeves, J. N., Sambruna, R. M., Braito, V., \& Eracleous, M. 2009a, ApJL, 702, L187

Reynolds, C. S. 1997, MNRAS, 286, 513

Romanova, M. M., Ustyugova, G. V., Koldoba, A. V., \& Lovelace, R. V. E. 2009, MNRAS, 399, 1802

Terashima, Y. 2010, in AIP Conf. Proc. 1248, X-ray Astronomy 2009 (Melville, NY: AIP), 337

Tombesi, F., Cappi, M., Reeves, J. N., \& Braito, V. 2012a, MNRAS, 422, L1

Tombesi, F., Cappi, M., Reeves, J. N., et al. 2010a, A\&A, 521, 57 
Tombesi, F., Cappi, M., Reeves, J. N., et al. 2011a, ApJ, 742, 44

Tombesi, F., Cappi, M., Reeves, J. N., et al. 2012b, MNRAS, 430, 1102 (T12b)

Tombesi, F., Sambruna, R. M., Marscher, A. P., et al. 2012c, MNRAS, 424, 754 (T12c)

Tombesi, F., Sambruna, R. M., Reeves, J. N., Reynolds, C. S., \& Braito, V. 2011b, MNRAS, 418, L89

Tombesi, F., Sambruna, R. M., Reeves, J. N., et al. 2010b, ApJ, 719, 700
Torresi, E., Grandi, P., Costantini, E., \& Palumbo, G. G. C. 2012, MNRAS, 419,321

Torresi, E., Grandi, P., Longinotti, A. L., et al. 2010, MNRAS, 401, L10

Tueller, J., Mushotzky, R. F., Barthelmy, S., et al. 2008, ApJ, 681, 113

Turner, T. J., \& Miller, L. 2009, A\&ARv, 17, 47

Vlahakis, N., \& Königl, A. 2003, ApJ, 596, 1080

Vlahakis, N., Tsinganos, K., Sauty, C., \& Trussoni, E. 2000, MNRAS, 318, 417 